\title{
APROXIMACIÓN INTERPRETATIVA A DETERMinANTES DE LA ViOLENCIA HOMiCidA EN CARTAGENA DE INDIAS DURANTE 2006
}

\author{
POr: FREDI GOYENECHE GONZÁLEZ
}

\section{RESUMEN}

Presentamos a continuación unas reflexiones interpretativas sobre el comportamiento anual de las muertes por homicidios durante 2006. Este documento trabaja con las cifras condensadas y consolidadas del Informe de Muertes por Causa Externa (MCE) 2006, que incluyen además de los homicidios, las Muertes no Intencionales (MNI), las Muertes en Accidentes de Tránsito (MAT) y los Suicidios. Estas cuatro variables, que conforman el concepto de MCE, son las que nos dan la idea de muertes violentas y obvio es pensar que mientras mas bajas sean estas, mejor debería ser la calidad de vida de los habitantes y visitantes del territorio en donde se estén haciendo las observaciones.

La intención de este documento es entonces aproximarnos a explicaciones de carácter teórico y académico que contribuyan a la comprensión no solo de las cifras como resultado, sino igualmente, al evidente crecimiento de estas en el contexto económico, político y social en que se producen.

\section{Palabras Claves: La violencia asesina, causas de la violencia urbana, estrategias de apropiación.}

\section{ABSTRACT}

The following work presents some interpretative reflections on the annual range of deaths caused by murders during the year 2006. This document works on the condensed and consolidated numbers in the report of Deaths Caused by External Factor (MCE) of 2006, which include, besides the murders, the undeliberate Deaths (MNI) the Deaths Caused by Car Accident (MAT) and the Suicides. These fourth variables which form the MCE concept are those which give us the idea of violent deaths and it is obvious to think that the lower these are the better should be the inhabitants and visitors quality of life of the territory where the observations 
are carrying on.

The intention of this document is then, to approach to theoretical and academic explanations which contribute to the comprehension of the numbers not only as a result but to the evident increase of these ones in the economical, political and social context where they are being produced.

\section{Key Words: The murdering violence, causes of the urban violence, appropriation strategies.}

\section{Contexto General de los Homicidios en Cartagena}

1 acerle seguimiento y análisis a las cifras de las muertes no intencionales, como son todas las ocurridas por accidentes diferentes a las de tránsito, por ejemplo las muertes por ahogamiento mostraría la connotación que esa información tiene para la vida económica local de la ciudad por su condición turística. Conocer cuántas victimas mortales ocurren por accidentes laborales, ilustraría sobre la seguridad con la que las empresas desarrollan sus actividades productivas.

Conocer el número de suicidios que se dan en el año y compararlos en una serie de tiempo puede ser una forma de dar piso a diagnósticos sobre la satisfacción o insatisfacción personal con la calidad de vida de una población. Pero en particular, y es de lo que vamos a ocuparnos, hacerle seguimiento a la frecuencia de homicidios en la ciudad determinando elementos caracterizantes de sus ocurrencias, es tener los insumos técnicos que nos permitan de forma mas acertada el diseño de políticas públicas tendientes a enfrentar la problemática del irrespeto a la vida complementariamente con la generación de un espacio de convivencia y armonía ciudadana.

En atención a esto, es absolutamente necesario hacer un seguimiento constante y riguroso del comportamiento tanto de la cifra que arroja la sumatoria de sus variables en conjunto, como el comportamiento singular de cada una de estas.

El total de homicidios ocurridos en Cartagena durante el año 2006 es de 275 casos que es 21.7 \% mayor a los 226 registrados el año anterior. Esta cifra se traduce en una tasa de homicidios para la ciudad de 30.7 homicidios por cada cien mil habitantes, pccmh, que es superior a la tasa de homicidios de Bogota, que por ejemplo, para este mismo año se situó en 18.7 pccmh. La cifra de este año se constituye en la segunda más alta de los últimos 12 años después de los 
293 casos registrados en 2003.

Gráfico 1

Los Homicidios en Cartagena por Meses del Año

$2005-2006$

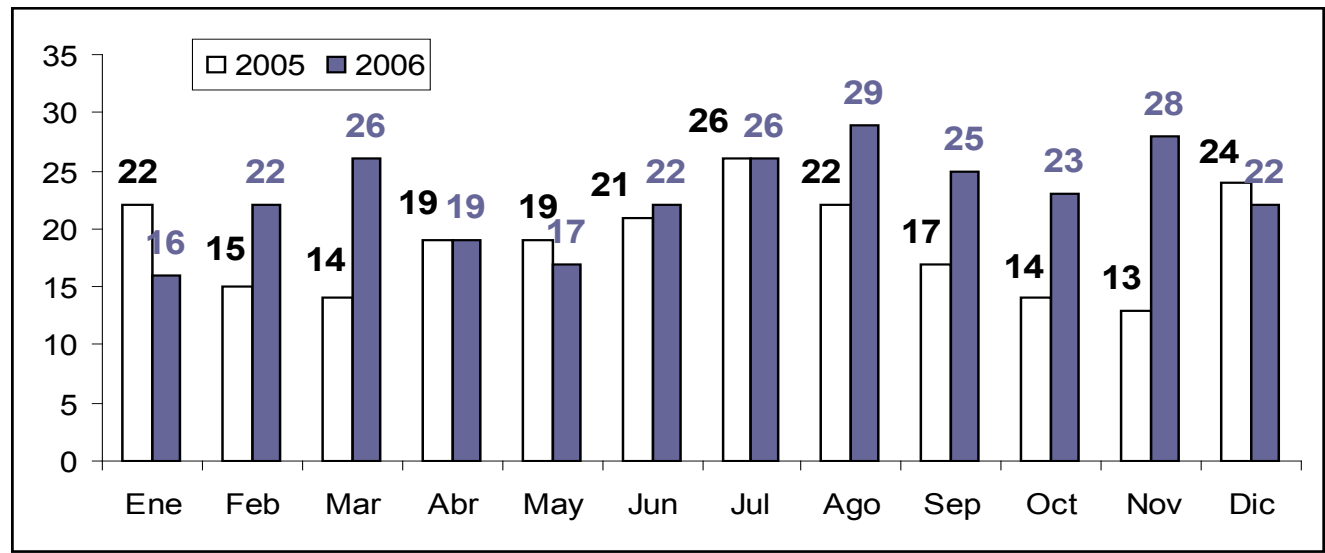

Aunque en ambos diciembres las cifras son relativamente altas, y que en igual sentido cursan las cifras en enero, de la desagregación y territorializacion de los homicidios, no se puede afirmar relaciones entre estos meses, el flujo turístico y la ocurrencia de estos delitos, tal como lo demuestra el trabajo de investigación "Contexto económico, político y social de las Muertes por Causa Externa en Cartagena de Indias, temporadas turísticas 1.995-2006" desarrollado bajo la dirección del COSED por los pasantes Oswaldo Mármol y Jerry Cuesta de la Universidad de Cartagena.

Gráfico 2

Los Homicidios en Cartagena por Días de la Semana $2005-2006$

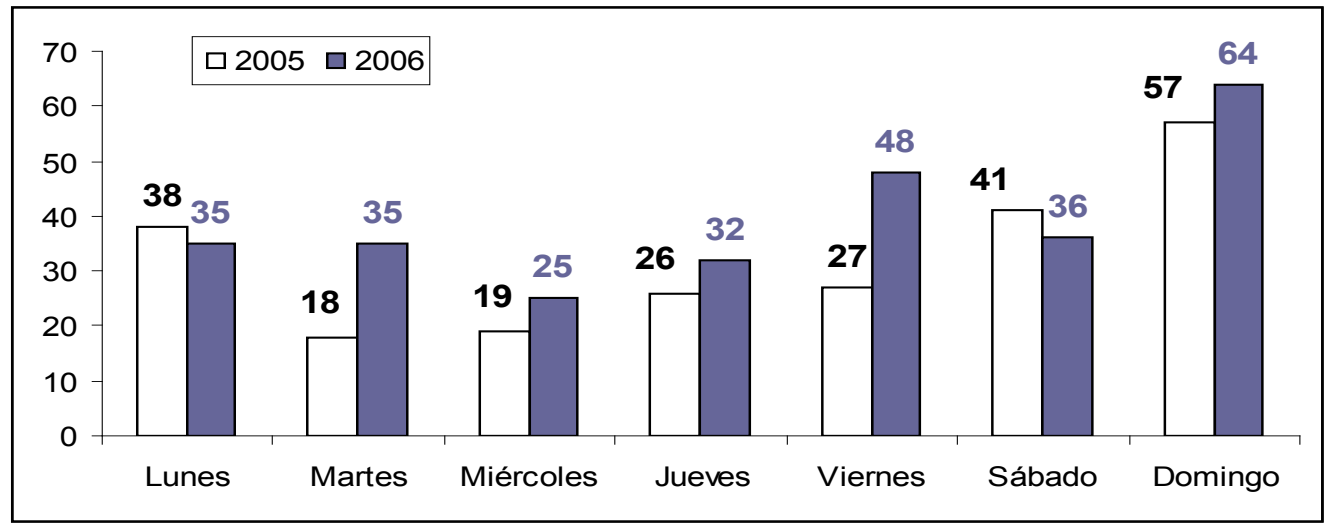

Palobrano. 8 Agosto/2007 
Los domingos, día de descanso y de actividades relacionadas generalmente con la familia y la lúdica, registra de lejos la mayor frecuencia de ocurrencia de homicidios observados por los días de la semana. Un poco mas del $25 \%$ de los homicidios ocurridos este año fueron en días domingos, 69 casos. Los días mas "seguros" durante 2006 de acuerdo al número de homicidios ocurridos en los diferentes días de la semana fueron los miércoles que registran la menor cifra, 25 casos en este año.

Esta situación tiene una potente afectación de la vida social de los ciudadanos en el disfrute del tiempo libre, desmejorando sin duda la calidad de vida de quienes percibirían este aspecto como limitante a la propia libertad individual en sus derechos a la seguridad en el sentido como lo plantea Amartya Sen cuando afirma que "en la medida en que el desarrollo tiene que ver con el logro de una vida 'vida mejor', habría que enfatizar la naturaleza - más que la forma - de la vida que lleva la gente". Si las oportunidades para el desarrollo de los individuos son radical y evidentemente distintas en términos muy desiguales, la propia libertad individual se encuentra restringida.

Entre los homicidios que ocurrieron este año en los días domingos, son significativos los ejecutados por sicarios materializados en la casa de la victima, lo cual agrisa mas la relación de descanso, hogar y homicidios. Sin embargo, los homicidios, como en general es la ocurrencia de estos en el Distrito, se localizan recurrentemente en sitios, sectores y barrios donde como ya se ha dicho guardan características socioeconómicas similares que determinan grados altos de pobreza y aun de indigencia. Es decir, el domingo se torna peligroso para los pobres.

La delincuencia organizada, dentro de la cual se ubican los homicidios por hurto, por sicariato y ajuste de cuentas, resulta ser la de mayor frecuencia en todo el 2006 reportando 146 de los casos, cifra superior en 25 casos a los reportados durante el 2005. Este aspecto podría fundamentar análisis de interesantes argumentaciones que considerarían frágil la relación entre pobreza y homicidios y más bien valorarían aspectos vinculados a racionalidades económicas del tipo de costo-beneficio como lo plantea Gary Becker. Es decir, que la impunidad entendida como perspectiva de costos para el antisocial se convertiría en una explicación más ajustada al aumento de actos delincuenciales que pueden desencadenar las circunstancias en que se contextualizan los homicidios. 
Gráfico 3

Los Homicidios en Cartagena por Contexto del Hecho

$2005-2006$

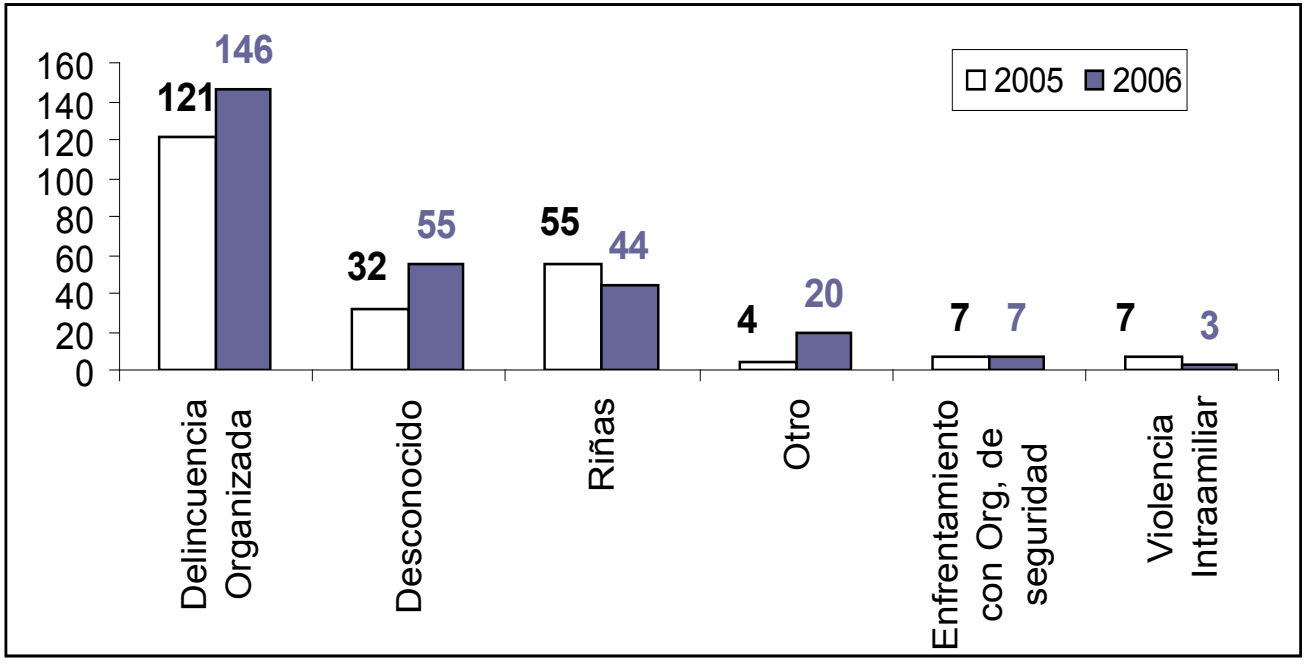

Las riñas representaron en el 2006 el segundo contexto de mayor frecuencia con 44 casos en total, cifra esta que connota una fragilidad del relacionamiento social para la resolución de conflictos; teniendo en cuenta el contexto socioeconómico, y la educación como elementos concomitantes a estos comportamientos. El contexto desconocido reporta 55 casos.

En este año, como en el anterior, se reportan 7 casos de personas que murieron en enfrentamiento con organismos de seguridad. Sin embargo, estas son igualmente muertes violentas, aunque estas muertes deberían diferenciarse de las que efectivamente son consecuencias del uso ilegitimo de la fuerza para quitar la vida, es decir el homicidio legalmente definido. Habría que complementar que estos casos no son superior al 2.5 \% del total del 2006 y del 3 \% para el 2005. La Violencia Intrafamiliar disminuyó la aportación de casos de homicidios este año con referencia al anterior

\section{Contexto Específico de los Homicidios en Cartagena}

La gráfica 4 permite observar que de los 6 barrios de mayor número de homicidios durante este año, 5 presentaron aumento en relación a las cifras registradas durante 2005 y solo 1 presentó una disminución. Olaya Herrera es el barrio de mayor número de homicidios este año con 28, 18.2 \% del total de homicidios registrados en la ciudad; le sigue El Pozon donde ocurrieron 21 casos 
de homicidios; el barrio Chino fue escenario de 13 casos y en el Bosque fueron registrados 12 homicidios. 74 homicidios acumularon estos 4 barrios durante el año 2006 es decir, el 27 \% del total de los ocurridos en todo el Distrito.

\section{Gráfico 4}

Los Homicidios en Cartagena por Barrios de Mayor Frecuencia

$2005-2006$

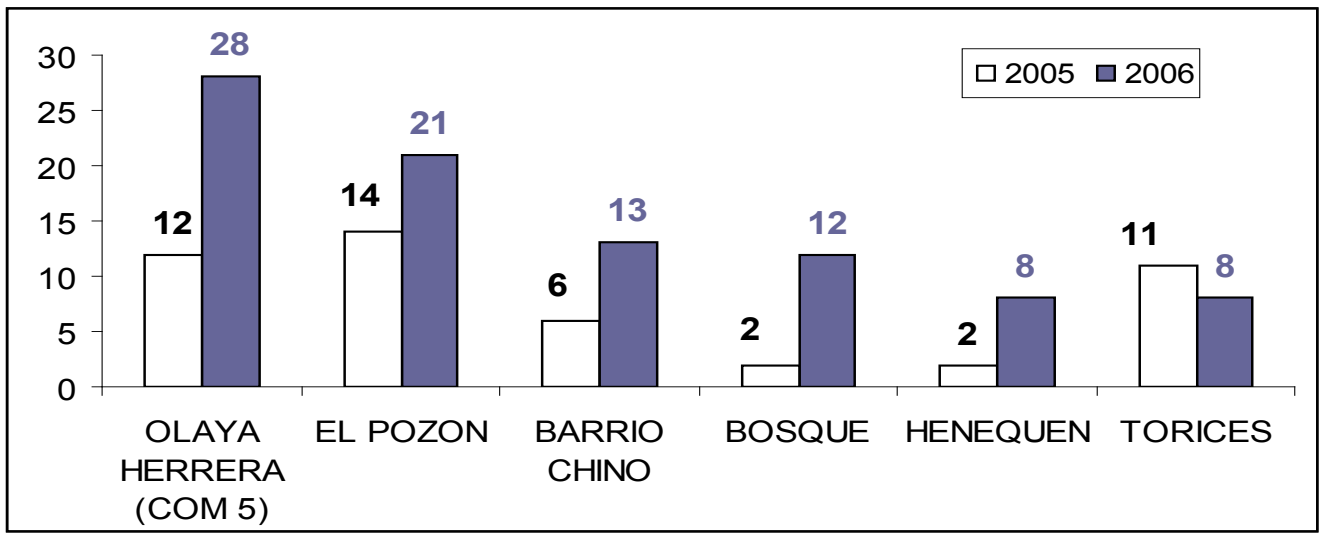

Estos barrios son caracterizadamente de estrato socioeconómico predominante de nivel 1. Los aumentos en barrios como El Pozon, barrio Chino, El Bosque, Henequen y Los Alpes deben ser analizados cuidadosamente teniendo en cuenta variables complementarias que contribuyan a acotar la comprensión del fenómeno.

\section{Gráfico 5}

Barrios con Mayor Numero de Homicidios a sus Residentes 2006

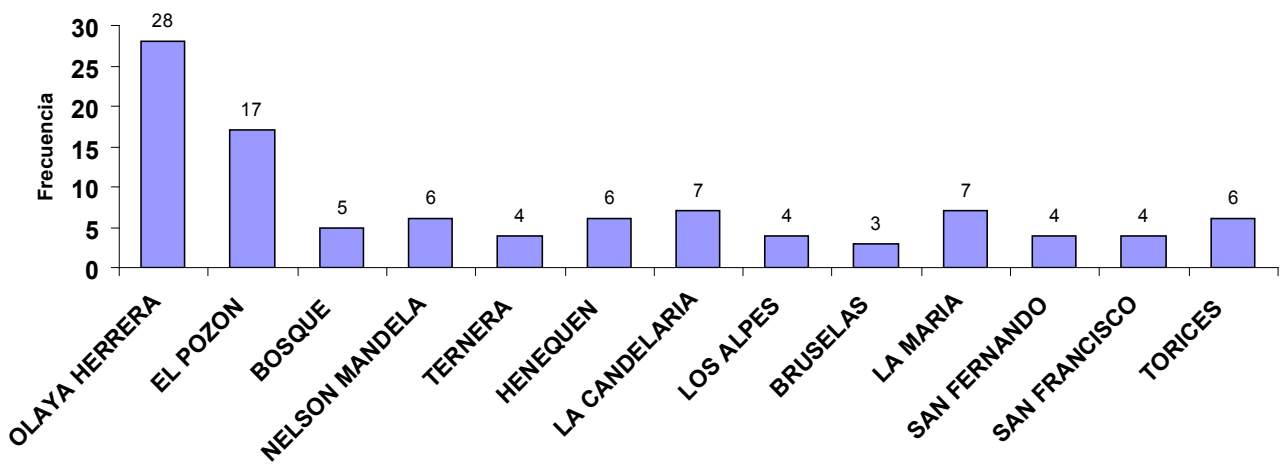


El barrio Olaya Herrera figura como el barrio de mayor número de homicidios contra sus residentes, 28 casos. Es también con el mismo número el barrio de más homicidios durante este año en el Distrito como quedó ya dicho anteriormente.

De los 275 homicidios del año 2006, 150 tuvieron como victimas a personas que residían en el mismo barrio donde se ejecutó el hecho, 54.4 \% del total. En barrios diferentes a los de residencias de las victimas se contabilizaron este año 80 casos, 29 \% del total de homicidios del año. $2.9 \%$ de las victimas fueron personas que no residían en la ciudad, estaban en Cartagena en condiciones de visitantes por diversas causas.

Sin embargo, debe afirmarse que los 36 casos en que no se pudo determinar con certeza la procedencia o residencia de la victima, es una cifra muy alta que le quita consistencia a la labor analítica.

Tabla 1

Los Homicidios en Cartagena. Clase de Lugar del Hecho $2005-2006$

\begin{tabular}{|c|c|c|}
\hline CLASE DE LUGAR & Frecuencia & Porcentaje \\
\hline CASA & 46 & 16,7 \\
\hline ESPACIO PUBLICO & 175 & 63,6 \\
\hline FINCA O CAMPO ABIERTO & 7 & 2,5 \\
\hline BAR O SIMILARES & 18 & 6,5 \\
\hline CARCEL & 3 & 1,1 \\
\hline LUGAR DE TRABAJO & 3 & 1,1 \\
\hline OTRO LUGAR & 17 & 6,2 \\
\hline NO SE SABE & 1 & 4 \\
\hline SIN DATO & 5 & 1,8 \\
\hline TOTAL & 275 & 100,0 \\
\hline
\end{tabular}

El espacio público fue el escenario mas frecuente de homicidios en el Distrito de Cartagena. El 64 \% de estos, 175 casos, ocurrieron en estos; este aspecto correspondería a la interpretación de la percepción ciudadana de peligro en las calles de la ciudad como se puede corroborar de los resultados de la Encuesta ciudadana 2006 de Ipsos-Napoleón Franco para "Cartagena Como Vamos" que muestra que un $61 \%$ de los encuestados percibe un desmejoramiento de la seguridad de la ciudad en 2006.

El segundo lugar de mayor frecuencia, 46 casos, fue paradójicamente la casa, lugar de tranquilidad por definición social, casi el 17 \% del total, fuertemente afectado este dato por los sicariatos que sumaron 104 victimas en este año. 
Los homicidios ocurridos en la casa tienen por característica común el de estar localizadas, con pocas excepciones, en barrios de nivel 1, incluido barrios como Olaya Herrera, El Pozon, Nelson Mandela. Contrario a lo anterior y a lo esperado, los homicidios ocurridos en bares o similares fueron menos de un tercio de los que se presentaron en las casas, ascendiendo este año a 18 casos, concentrándose todos en lugares de diversión localizados por fuera del Centro Histórico, lo cual contrasta la seguridad de los sitios de diversión de acuerdo a su localización territorial.

En general, un diagnostico de primera mirada daría cuenta de las implicaciones sociales de los homicidios y se agrava mas si tenemos en cuenta el numero de victimas identificadas como estudiantes que además tienen la condición de ser habitantes de barrios de condiciones precarias. La coincidencia de los homicidios y la pobreza en todas sus expresiones son los elementos que nos sirven para aproximarnos a un concepto de pobreza más completo considerando aspectos como la salud, educación o la vivienda.

Dos de las tres de las localidades en que se encuentra organizado administrativamente el Distrito mostraron aumento de los homicidios durante 2006 comparados con el 2005.

La Localidad Industrial de la Bahía registró disminución en el número de homicidios y es además la de menor número con 70 casos, $25.4 \%$ de los ocurridos este año, 6 menos que en 2005. Es de resaltar que el año anterior había sido la de mayor número de homicidios.

Dos de las tres de las localidades en que se encuentra organizado administrativamente el Distrito mostraron aumento de los homicidios durante 2006 comparados con el 2005.

La Localidad Industrial de la Bahía registró disminución en el número de homicidios y es además la de menor número con 70 casos, 25.4 \% de los ocurridos este año, 6 menos que en 2005. Es de resaltar que el año anterior había sido la de mayor número de homicidios.

Esta localidad agrupa las comunas entre la unidad comunera $\mathrm{N}^{0} 11$ y la $\mathrm{N}^{\mathrm{o}}$ 15. Los barrios de estas comunas son heterogéneos en cuanto a composición socioeconómica aunque priman los estratos 1, 2 y 3 siendo la mas densificada la Unidad Comunera No 15 con 64.000 habitantes a septiembre de 2.003 de los 
cuales 33.332 son de estrato 1 y 20.789 son de estrato 2. Esta comuna registró 13 homicidios en los 15 barrios que la constituyen, de los cuales 8 se concentraron en el barrio Henequén y 3 en San Pedro Mártir.

\section{Gráfico 6}

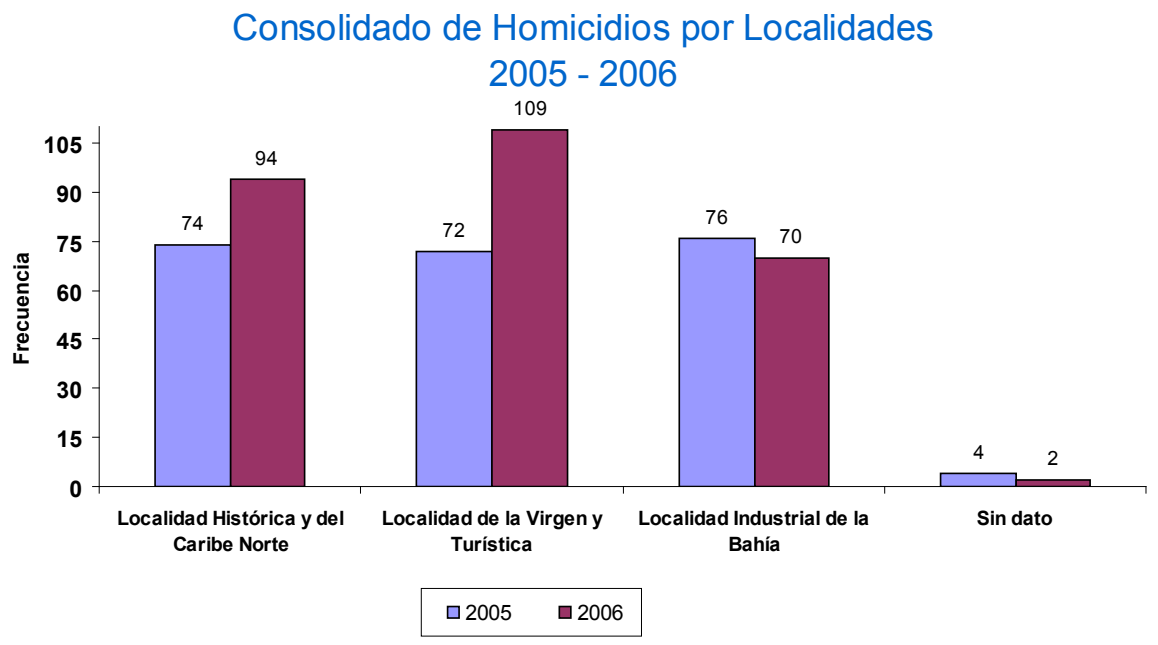

Las Unidades comuneras de gobierno de esta localidad que más frecuencia de homicidios presentaron fueron la $N^{0} 11$ y la $N^{0} 12$ con 15 y 17 casos respectivamente las cuales conjuntamente con la unidad $N^{\circ} 14$ y la $N^{\circ} 13$ ya mencionada concentran el 61.4 \% de los homicidios de la localidad. Particularme podemos señalar a los barrios Henequén, Ternera, San Fernando y Nelson Mandela que suman 27 homicidios, es decir, $38.5 \%$ de los homicidios de la localidad en estos 4 barrios sobre un total de 73 barrios que agrupa.

La Localidad de la Virgen y Turística tuvo un comportamiento contrario a la anterior, de ser la de menor número de casos en 2005 con 72, registró este año 109 casos que equivalen al $40 \%$ de los homicidios ocurridos durante 2.006 en la ciudad. Un crecimiento del 51.3 \% frente a los ocurridos durante 2005 en esta misma localidad. Este comportamiento es observable en cada comuna de su jurisdicción, localizándose en esta localidad la comuna No 5 que es la comuna de mayor numero de homicidios con 34 casos. Le sigue su comuna colindante, la comuna No 6 que arroja 27, estas dos comunas arropan a Olaya Herrera que se ha dicho tiene el mayor número por barrio con 28 casos.

Esta localidad la constituyen 34 barrios. Se presenta dificultad y confusión a la hora de establecer con precisión el barrio del hecho cuando este corresponde a Olaya Herrera dado que bajo este nombre figuran dos barrios, uno en la comuna 
No 5 y otro en la Comuna No 6, con continuidad territorial y claramente definidos territorialmente en el documento "Cartagena de Indias D.T. Y C. Localidades, territorio y población" Secretaria de Planeacion, Alcaldía Mayor de Cartagena, 2003. Si relacionáramos el total de homicidios en Olaya Herrera, los dos barrios que figuran con ese nombre, y que como dijimos son colindantes, con la población residente, resultaría una tasa de homicidios de 43.1 hpccmh, que es mayor que la tasa nacional y de ciudades como Bogotá, Medellín, Cali y Barranquilla, y mucho mayor también a la tasa total de la ciudad.

Por otra pare, la Localidad Histórica y del Caribe Norte tuvo también un aumento significativo en el número de homicidios ocurridos en su territorio al pasar de 74 casos en 2005 a 94 en 2006, un aumento del orden del 21.2 \%, 34 \% del total de homicidios ocurridos en la ciudad este año. Las Unidades comuneras que la constituyen van de la № 11 hasta la 15 y además cobija a Pasacaballos que es unidad comunera rural, el sector Membrillal y el sector de la variante Cartagena y Cordialidad. La comuna de mayor cantidad de homicidios de esta localidad es la No 12, comuna con promedio de estratificación socioeconómica de nivel 3, a la cual pertenecen los barrios El Socorro y Los Caracoles que registran 3 homicidios cada uno y El Campestre con 2 casos.

\section{Magnitud, Razones y Causas de la Violencia Urbana}

Simultáneamente con los análisis de las cifras, frecuencias, indicadores, tasas y demás elementos propios de este tipo de ejercicios, creemos pertinente, hacer algunos comentarios sobre otros, aspectos que articulados con los anteriores, nos ayudarían a aproximarnos a la comprensión de las causas de las muertes violentas en la ciudad y del fenómeno de la violencia relacionada particularmente con los homicidios. Los indicadores sobre pobreza, desempleo, desplazados forzados llegados a la ciudad y la distribución del ingreso, entre los más importantes, son datos que vistos conjuntamente con los indicadores de seguridad ciudadana nos ayudan a comprender la magnitud, razones y causas de la violencia urbana.

Desde tiempo atrás en nuestro país se ha asistido a un fuerte debate frente a diversas interpretaciones sobre los niveles de violencia y su relación para algunos y causalidad para otros, con variables tales como los niveles de pobreza y de desempleo o con la concentración del ingreso. Ciertamente este debate no se ha definido de forma inequívoca y menos definitiva. Tal vez la polarización interpretativa bajo metodologías y enfoques diferentes sea más la sintomatología de la incapacidad de poder precisar razones, relaciones y causalidades que no pueden 
comprender la complejidad del fenómeno de la violencia y quieran determinar de manera axiomática relaciones y causalidades que tienen importancias singulares mas no tienen el bálsamo tranquilizante de la generalidad.

Sin embargo, Cartagena ha experimentado un aumento de las Muertes por Causa Externa impulsada sobre todo por el peso que los homicidios tienen en la definición de las cifras de estas; el comportamiento aunque oscilante muestra tendencias crecientes. Cartagena conjuga varios aspectos que pudieran evidenciar conexiones con el comportamiento observado para los homicidios.

Uno de gran peso, tiene que ver con la alta tasa de desempleo de la ciudad que en los últimos 12 años se ha mantenido por encima de 2 dígitos, siendo en los 3 últimos años de los más altos entre las principales ciudades del país y siempre por encima del promedio nacional. En noviembre de 2006 la tasa de desempleo en el Distrito informada por el Dane se ubicó como la segunda mas alta del país con $16.7 \%$. A ello habría que añadirle que la tasa de subempleo ronda el $20 \%$ lo cual significa, como lo afirman Luís Zuleta y Lino Jaramillo en su documento para el Convenio Andrés Bello y la Corporación Centro Histórico de Cartagena de Indias "Cartagena de Indias, impacto económico de la Zona Histórica, 2006", que mas de la tercera parte de la población económicamente activa no obtienen ingresos u obtiene ingresos que no alcanzan siquiera la mitad del salario mínimo legal lo cual nos acerca a la percepción generalizada en círculos académicos en el sentido de que el comportamiento de la línea de pobreza esta estrechamente correlacionado con la tasa de desempleo.

Un trabajo de Adolfo Meisel y Luís A. Galvis, "El crecimiento económico de las ciudades colombianas y sus determinantes 1.973-1998", estimaba que el ingreso per capita real en el Distrito de Cartagena correspondía al 57\% del ingreso per capita real de una muestra de las 20 principales ciudades del país. Anotado lo anterior, vale observar igualmente que los barrios de mayores grados de informalidad tanto en lo laboral como en su cotidianidad económica, que presentan a sus habitantes mas vinculados a actividades y ocupaciones temporales que al empleo formal, registran también las mayores tasas de homicidios analizadas por barrios de la ciudad.

Dado que "la tasa de crecimiento promedio anual de la población de esta ciudad es superior a las tasas del departamento de Bolívar y de la Nación: para el año 2000 el país presenta un crecimiento anual de 1,9 por ciento, el departamento 2,5 y el Distrito 2,9. En el año 1995, la población de Cartagena 
era de 780.527; en 2000 aumentó a 902.005; las proyecciones al año 2015 estiman en 1.300.000 el número de personas", un aspecto que en esta situación contribuiría a agravar el cuadro de pobreza, sobre todo si se tiene en cuenta que "por grupos de edad, los menores de 6 años son el 15\% y los rangos de 7 a 15 años y de 16 a 24 representan cada uno un 18\%. Esta situación genera grandes retos en coberturas de educación, salud, recreación y una creciente necesidad de nuevos puestos de trabajo una vez ingrese esta población al mercado laboral. Estas dos ultimas, afirmaciones del informe final del documento "Formulación de las bases de la política social estratégica para Cartagena de Indias", Convenio Alcaldía de Cartagena de Indias y la Corporación Viva la Ciudadanía Bogotá, diciembre de 2003.

Por otra parte, a pesar de que la ciudad muestra en su presente grandes potencialidades de crecimiento económico, y que ha mostrado un satisfactorio crecimiento del PIB local, por encima del crecimiento del mismo a nivel nacional tanto en el total como en el per cápita, como lo demuestra "El calculo y análisis del crecimiento económico en el Distrito de Cartagena y su impacto en la calidad de vida, 1990-2000" del Departamento de Investigaciones Económicas de la Universidad de Cartagena, y se puede inferir de información de la Cámara de Comercio, esta nueva riqueza no se ve adecuadamente distribuida, desde la perspectiva de la equidad social, entre los actores económicos locales ni mucho menos en la participación de acuerdo a la composición social de la población residente.

Esto significaría que estamos en un proceso dinámico de concentración del ingreso o de distribución de la nueva riqueza generada, que es lo mismo, con una profundización de la polarización social de los habitantes de la ciudad. Tal vez estos aspectos articulados sean clave para la comprensión de la problemática. Seguramente pudiera parecer paradójico y contradictorio que sea en los periodos que algunos llaman bonanzas cuando es mas observable como se concentran los ingresos y la riqueza en segmentos de muy restringido acceso para el grueso de la sociedad y que por lo tanto se privilegia en la distribución de la riqueza.

Igualmente, los homicidios se concentran, como ya lo anotamos, en zonas que tienen características casi homogéneas de pobreza; A pesar de ello, vale agregar que instrumentos como el SISBEN utilizado con el objetivo concreto de focalizar los beneficiarios de subsidios dan cuenta de alrededor de 700.000 cartageneros que están identificados como niveles 1 y 2 , es decir, aplican para subsidios que por su naturaleza están destinados a los pobres, luego entonces tienen la 
condición de tal. Y esta población de acuerdo a este sencillo calculo estaría en el orden de aproximadamente el 70\% de la población local. Tal como lo afirma el profesor Efraín Cuadro en el trabajo dirigido para el DIES de la Universidad de Cartagena "Informe final de los posibles impactos generados por la celebración de los XX juegos Centroamericanos y del Caribe 2006 en Cartagena", la pobreza y la marginalidad impiden a un gran segmento de la población cartagenera ejercer derechos elementales de trabajo seguro y bien remunerado, alimentación, educación, atención medica, vivienda, recreación y deporte.

En estos espacios urbanos descritos donde repetidamente se localizan los espacios de la pobreza y los homicidios, otros elementos concomitantes a esta conceptualidad afloran en el déficit de vivienda que se expresa en el hacinamiento disociante del desarrollo familiar en términos de respeto por la dignidad individual y grupal. Citando el último informe de Cartagena como Vamos "Necesidades habitacionales, calidad de vida y competitividad en Cartagena"... "según las estimaciones del CENAC, el total de hogares en déficit en Cartagena a 2005 fue de 79.157, equivalentes al 35.9\% del total de hogares de la ciudad. De ellos, 48.609 se encontraron en déficit cualitativo, siendo la falta de espacio y de servicios públicos la principal carencia de los hogares", pero complementariamente con eso, "la mayor parte del déficit (70.2\%) se concentra en el estrato 1".

Pero además "en el $81.9 \%$ de los hogares en déficit, el jefe de hogar reporta ingresos mensuales inferiores a la mitad del valor del salario mínimo (SMLV =\$408.000 de 2005)." Una gran parte de esta población está ubicada sobre la Ciénega de la Virgen como parte de la localidad del mismo nombre, donde sus habitantes se perciben así mismos, el $58 \%$, como los mas pobres de las tres localidades resultado que arroja la encuesta de percepción 2006 adelantada por Ipsos Napoleón Franco para el grupo que financia Cartagena Como Vamos. Los homicidios están presentes en mayor número en la medida en que la pobreza se profundiza de forma inequívoca y evidente.

Un elemento adicional habría que agregar al escenario donde discurren su vida los residentes locales, es el flujo constante y creciente hacia la ciudad de desplazados forzados, producto del conflicto interno. Estudiosos del tema en el impacto que esto produce como, el investigador Amaranto Daniels en su más reciente ensayo, "Cartagena: Ciudad fragmentada", en el cual calcula en mas de 50.000 los desplazados ubicados en la ciudad, o trabajos menos recientes pero de gran aportación académica: "Operación Prolongada de Socorro y Recuperación. Cartagena 2000-2005", presentados por el profesor Sandro Jiménez de la 
Universidad San Buenaventura, muestran las características de estas victimas de la violencia y la potencialidad de violencia que pueden generar en su afán entendible de localización en la ciudad. Cartagena hasta el año 2005 según datos de la Red de Seguridad Social seccional Bolívar ha recibido cerca de 39.292 desplazados agrupados en 9362 familias, lo que representa el 2.2\% de las personas y el 2.3\% de los hogares desplazadas del país.

Cartagena se ubica como la sexta ciudad de Colombia con mayor número de desplazados después de Bogota, Medellín, Santa Marta, Sincelejo y Valledupar. Sin embargo, al relacionar este número de desplazados oficialmente reconocidos con la población establecida en el censo 2005 resulta Cartagena con una tasa de localización de 4.388 desplazados por cada cien mil habitantes mientras que Medellín y Bogota tienen tasas de 3.790 y 1.637 respectivamente (todos los cálculos del autor).

Hasta el 29 de Septiembre del 2003, los lugares donde se concentraba el mayor número de desplazados eran los barrios Nelson Mandela que albergaba el 13\% de estos, El Pozon con el 11\%, Olaya Herrera con el 7\% y San José de los Campanos con el 2\%. Estos barrios donde se concentra la población desplazada, se caracterizan por ser lugares con niveles altos de pobreza, carencia en servicios públicos como agua potable y alcantarillado, altos niveles de informalidad laboral y riesgos ambientales para sus habitantes tal como lo ilustra Informe de Coyuntura Social, "Indicadores Sociales de Cartagena, II Semestre 2003" de abril de 2004 publicado por la Cámara de Comercio; además, registran el mayor número de homicidios que se presentan en la ciudad (caso de El Pozon, Olaya Herrera y Nelson Mandela). Una trágica paradoja, en la cual los pobres que vienen desplazados por la violencia política y la delincuencia organizada del sector rural se enfrentan a los pobres históricos de la ciudad por los escasos espacios y recursos que el Distrito destina a su atención.

Finalmente la ciudad podría decirse, sufre un doble proceso frente al desplazamiento. Por una parte es receptora importante de desplazados forzosos con su grave carga de demandas de inversión pública y por el otro, el periodo especulativo ha provocado un desplazamiento interno de los habitantes de barrios cuyo suelo se ha valorizado de forma trágicamente excluyente, casos de San Diego, El Centro y Getsemani que fueron barrios de una heterogeneidad socioeconómica trastocada en una particular clase media caribeña, barrios que en la actualidad están transformando su naturaleza de uso residencial por usos en los que se privilegia la explotación económica, mientras sus antiguos residentes 
se relocalizan en barrios de la periferia del centro amurallado.

\section{Estrategias de Apropiación de la Ciudad}

Cartagena sufre una profunda renovación en el uso del área urbana sin precedentes, vinculada al uso del suelo presionado por el momento económico, lo que produce una fuerte especulación inmobiliaria que hace caras las tierras y por lo tanto escasa la destinada para la construcción de vivienda de interés social ahora y en el futuro próximo, se confunde por el vértigo de los cambios con el mismo presente.

Esos cuatro elementos apretadamente mencionados giran en torno a un núcleo constituido por la ciudad patrimonio cultural, la ciudad turística y la ciudad histórica que se combinan en una singularidad atractiva para los ricos que convierten a la ciudad vieja en un bien para el uso, que es una forma estilizada de decir que la ciudad se convierte ella misma en una mercancía. No es casual que esta ciudad halla sido la paradigmática si se habla de mostrar la aplicación rigurosa de los mandamientos del Consenso de Washington, el neoliberalismo exacerbado del último cuarto de siglo anterior.

Cartagena fue la primera ciudad del país en privatizar sus servicios públicos en su totalidad y paulatinamente sus espacios públicos también avanzan en el mismo sentido. Las plazas públicas tan cercanas a la esencia de la naturaleza de lo público y de ejercicio de la ciudadanía, al mejor decir de García Canclini, expresan de forma dramática la metamorfosis del ciudadano local; este ya no puede disfrutar esos espacios en su condición de ciudadano, solo lo puede hacer en la condición de consumidor dado que estos espacios públicos están en usufructo de empresarios que condicionan su permanencia en estas plazas solo a los consumidores de sus ofertas. Solo el poder de compra, el poder adquisitivo le dará al ciudadano de este tiempo el derecho al uso de lo que fue colectivo.

Pero Cartagena es también atractiva para los pobres que se toman la ciudad y su espacio público para la construcción de sus viviendas informalizadas o mediadas por la invasión de lotes sin servicios y la construcción pirata. La atracción de esa combinación de elementos mencionados mas arriba surte el efecto de obnubilar los ojos ansiosos y las esperanzas reinventadas de los cientos de colombianos que miran hacia la ciudad y orientan sus pasos en busca de la oportunidad de vida que le insuflan las luces del centro histórico, turístico y monumental de Cartagena de Indias D. T. y C.

Palobrano. 8 Agosto/2007 
El resultado de esta combinación de aspectos económicos y sociales no ha sido benéfico para la ciudad. Tal como lo hemos anotado, las mayores frecuencias de homicidios se presentan en los espacios de la pobreza. La coincidencia territorial de violencia y de pobreza es visible al analizar los mapas de territorializacion de la violencia en Cartagena en sus formas de homicidios, de delitos sexuales o de violencia intrafamiliar.

\section{BIBLIOGRAFIA}

ALCALDÍA DE CARTAGENA DE INDIAS; CORPORACIÓN VIVA LA CIUDADANÍA. Formulación de las bases de la política social estratégica para Cartagena de Indias. Convenio. Bogotá.

CARTAGENA COMO VAMOS. (2006); Una mirada integral a la pobreza en Cartagena. Evaluación de calidad de vida. Encuesta de Percepción Ciudadana 2005 y 2006.

CONVENIO ANDRÉS BELLO; Cartagena de Indias, impacto económico de la Zona Histórica. Corporación Centro Histórico de Cartagena de Indias; 2006,

COSED. Informe anual de Muertes por Causa Externa, 2006. Centro de Observación y Seguimiento del Delito. Cartagena, enero 2007.

CUADRO, Efraín y otros. (2006); Informe final de los posibles impactos generados por la celebración de los XX juegos Centroamericanos y del Caribe 2006 en Cartagena. Departamento de Investigaciones Económicas y Sociales, (DIES), Universidad de Cartagena.

DANIELS, Amaranto. (2006); El desplazamiento en Cartagena: entre la invisibilidad y la incertidumbre. Instituto internacional del Caribe, Universidad de Cartagena

(2007); Cartagena: Ciudad fragmentada. Centro de Estudios Internacionales del Caribe. Universidad de Cartagena.

GARCÍA CANCLINI, Néstor. (1995); Consumidores y ciudadanos - Conflictos Multiculturales. Editora Grijalbo, Méjico.

GOYENECHE, Fredi; MÁRMOL, Oswaldo; PARDO, Jerry. (2007); Contexto económico, político y social de las Muertes por Causa Externa. Cartagena. Temporadas turísticas, 1.994-2.006, Centro de Seguimiento y Observación del Delito, COSED. Cartagena.

JIMÉNEZ, Sandro. (2006); Operación Prolongada de Socorro y Recuperación. Cartagena 2000-2005. Universidad de San Buenaventura. Escuela Latinoamericano de cooperación y desarrollo. Cartagena. 
MEISEL, Adolfo; GÁLVEZ, Luís. (2000); El crecimiento económico de las ciudades colombianas y sus determinantes 1.973-1998. Centro de Investigaciones del Caribe Colombiano, Banco de la Republica. Cartagena 2000.

RED DE SOLIDARIDAD SOCIAL, BOĹ́VAR. (2005); Base de datos.

SECRETARIA DE PLANEACION. (2003); Cartagena de Indias D.T. y C. Localidades, territorio y población. Alcaldía Mayor de Cartagena.

SEN, Amartya. (1985); Desarrollo: Ahora, ¿hacia dónde?". Original en: Economic Journal, Vol. 93, No 372, diciembre 1983.

GOYENECHE, Fredi; MÁRMOL, Oswaldo; PARDO, Jerry. (2007); Contexto económico, político y social de las Muertes por Causa Externa. Cartagena. Temporadas turísticas, 1.994-2.006, Centro de Seguimiento y Observación del Delito, COSED. Cartagena.

\section{BIOGRAFIA FREDI GOYENECHE GONZÁLEZ}

Economista, especialista en Planeación del Desarrolloy en Gestión Pública. Ex-director de la ESAP Regional Bolívar, Docente Facultad de Ciencias Económicas, Universidad de Cartagena y Universidad Tecnológica de Bolívar. Director Centro de Observación y Seguimiento del Delito COSED. 